\title{
Development of Environmentally Friendly Modified Fe-PAN Fibrous Catalyst and Its Application in Degradation of Dye
}

\author{
Jinna Wu, Juanzhi Zhao, Fang Du \& Zhenbang Han \\ Division of Textile Chemistry \& Ecology, School of Textiles, Tianjin Polytechnic University \\ Tianjin 300160, China \\ E-mail: wujinna2007@163.com
}

\begin{abstract}
Modified Fe-PAN fibrous catalyst (Fe-AO-PAN) was formulated at the room temperature by the reaction of amidoxime (AO) modified polyacrylonitrile (PAN) fiber (AO-PAN) with $\mathrm{FeCl}_{3}$, which was in turn used as an environmentally friendly heterogeneous photocatalyst in the oxidative degradation reaction of the standard target dye Rhodamine $\mathrm{B}$ in the presence of hydrogen peroxide. This paper investigated the influences of the iron ion concentration in solution $\left(C_{\mathrm{Fe}}\right)$ and reaction temperature to the iron ion content in Fe-AO-PAN catalyst $\left(C_{\mathrm{Fe}-\mathrm{AO}-\mathrm{PAN}}\right)$; studied the effects of $C_{\mathrm{Fe}-\mathrm{AO}-\mathrm{PAN}}$, $\mathrm{pH}$, intensity of light irradiation and etc. on the decoloration rate of the dye; and analyzed the oxidative degradation procedure of the dye by ultraviolet-visible light spectrum analysis. The results indicate that $C_{\mathrm{Fe}-\mathrm{AO}-\mathrm{PAN}}$ increases with the rise of $C_{\mathrm{Fe}}$ or reaction temperature; the decoloration rate of Rhodamine $\mathrm{B}$ increases with the rise of $C_{\mathrm{Fe}-\mathrm{AO}-\mathrm{PAN}}$ or radiation intensity, as well as the decrease of initial concentration of the dye; and Fe-AO-PAN shows higher catalytic activity in the solution of $\mathrm{pH}$ less than or equal to 6.0, however lower catalytic activity in a strongly basic medium. In addition, ultraviolet-visible light spectrum analysis proves that Fe-AO-PAN can effectively catalyze the break of azo bond and aromatic ring in the dye molecular, but can not be reutilized efficiently.
\end{abstract}

Keywords: Modified PAN, Environmentally friendly heterogeneous photocatalyst, Rhodamine B, Degradation of dye

\section{Introduction}

At present, the dyeing process wastewater is one of the hard-to-treat industrial wastewaters in China. With the rapid development of the dye and dyeing process industry, the resulted pollution to the environment is on the rise. However, it is hard to process numerous and jumbled dye wastewaters of complex constituents, high chromaticity and high toxicity by physiochemical technology and biotechnology. Now, the widely applied homogeneous photo-assisted Fenton oxidation process has great advantages in treating the hard-to-degrade, harmful and toxic pollutants, and is being taken more seriously by professionals. Homogeneous reactions are chemical reactions in which the reactants, products and catalyst are in the same phase. All reactants and catalysts in the reaction contact with each other sufficiently, and appear higher activity and selectivity, but it is hard to reutilize the catalyst. In homogeneous Fenton reaction, $\mathrm{pH}$ is limited in a small range of 2.0 5.5, and large amount of remaining iron ion (as catalyst) in the solution leads to the secondary pollution which increases the process cost of wastewater. Therefore, it is necessary to improve the Fenton reaction system to separate iron ion from the homogenous solution, then absorb iron ion onto the carrier. The formed heterogeneous Fenton reaction system lowers the secondary pollution and the process cost by reutilizing iron ion. In one word, with its strong degradation ability, no secondary pollution, easy-to-recycle catalyst and etc., heterogeneous Fenton technology becomes a hot topic in the environmental protection research.

This paper studied on the preparation of Fe-AO-PAN catalyst applicable to the heterogeneous Fenton system, and investigated its catalytic degradation on the standard target dye Rhodamine $\mathrm{B}$. The results have important and instructive significances to the further studies on the catalytic property of the environmentally friendly Fe-PAN fibrous catalyst, decrease of the environment pollution from dye, as well as realization of the sustainable development.

\section{Experiment}

\subsection{Reagent and dye}

PAN yarn, $\mathrm{NH}_{2} \mathrm{OH} \cdot \mathrm{HCl}, \mathrm{FeCl}_{3} \cdot 6 \mathrm{H}_{2} \mathrm{O}, \mathrm{NaOH}$ and $\mathrm{H}_{2} \mathrm{O}_{2}$, commercial analytical reagents; Rhodamine $\mathrm{B}$, a pure dye sample provided by Tianjin Sanhuan Chemical Co., Ltd., whose chemical structure is shown in figure 1. 


\subsection{Experimental instrument}

723 spectrophotometer, from Shanghai Analytical Instrument Co., Ltd; UV-PC2401 ultraviolet-visible light spectrophotometer, from Shimadzu Corporation of Japan; PH-25 digital pH meter, from Shanghai Precision \& Scientific Instrument Co., Ltd.; UV-A radiometer and FZ-A radiometer, both from Photoelectric Instrument Factory of Beijing Normal University; AL204 electronic analytical balance, from Mettler-Toledo (Shanghai) Instrument Co., Ltd.; 400 W high-voltage mercury lamp, from Osram (foshan) Co., Ltd.; HZ-1 magnetic stirrer, from Shanghai Secondary Analytical Instrument Co., Ltd.; and water-cooled photochemical reactor, Chinese Utility Model Patents (No. 03275610.0).

\subsection{Preparation of Fe-AO-PAN catalyst}

\subsubsection{Modification of PAN fiber}

Put accurately weighed PAN yarn into a certain concentration of aqueous solution of $\mathrm{NaOH}$ and $\mathrm{NH} \mathrm{H}_{2} \mathrm{OH} \cdot \mathrm{HCl}$ to obtain a mixture of $\mathrm{PAN}, \mathrm{NH}_{2} \mathrm{OH} \cdot \mathrm{HCl}$ and $\mathrm{NaOH}$ in the molar ratio of 1.0:1.25:0.75. Stir for 1.5 hours at $(70 \pm 1){ }^{\circ} \mathrm{C}$ under the conditions of $\mathrm{pH}$ 5.5 6.0 to get AO-PAN fiber, take it out and wash with distilled water until $\mathrm{pH}$ is close to neutral, then dry it for further use. The reaction equation is as following formula (1).

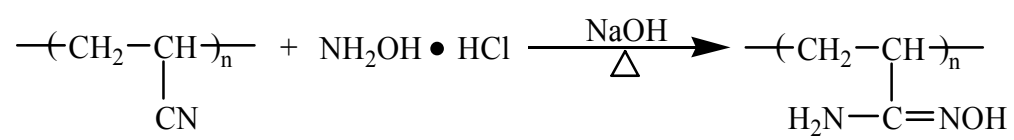

\subsubsection{Coordination reaction}

Dip AO-PAN fiber into a given concentration of $\mathrm{FeCl}_{3}$ solution, mix on the magnetic stirrer at $\mathrm{pH} 1.5 \sim 2.5 \mathrm{for} 6 \mathrm{~h}$, then repeatedly wash with distilled water until no detectable chloride ion. The obtained chocolate-brown yarn is dried to get Fe-AO-PAN catalyst. The reaction equation is as following formula (2).

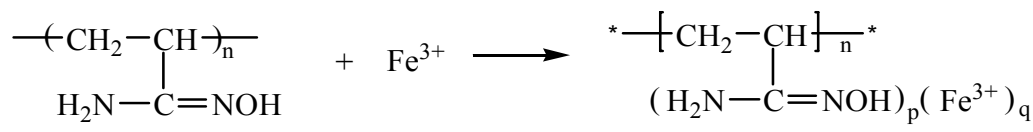

2.4 Determination of $\mathrm{Fe}^{3+}$ content $\left(C_{\mathrm{Fe}-A O-P A N}\right)$

Add a certain quantity of Fe-AO-PAN catalyst into $20 \mathrm{~mL} 2.0 \mathrm{~mol} / \mathrm{L} \mathrm{H}_{2} \mathrm{SO}_{4}$ solution. It is observed that chocolate-brown of the solution gradually turns into white after about $6 \mathrm{~h}$, which indicates that iron ion therein is completely desorbed from catalyst. Determine the concentration of iron ion in the desorption solution by Phenanthroline Method, and calculate $C_{\mathrm{Fe}-\mathrm{AO}-\mathrm{PAN}}$ per unit mass.

\subsection{Degradation process of dye}

Photocatalytic degradation of the dye is carried out in a water-cooled photochemical reactor which is composed of light source (high-voltage mercury lamp and UV lamp), temperature-controlled magnetic stirrer, reactor (special beaker), glass rack and etc. Add $100 \mathrm{~mL}$ mixed solution of $0.02 \mathrm{mmol} / \mathrm{L}$ dye (unless otherwise specified) and $3.0 \mathrm{mmol} / \mathrm{L} \mathrm{H}_{2} \mathrm{O}_{2}$ into the reactor, regulate to a certain $\mathrm{pH}$, then dip a certain quantity of Fe-AO-PAN catalyst (fixed by winding on the surface of glass rack) into the solution, react at $20 \pm 1{ }^{\circ} \mathrm{C}$ in the water-cooled photochemical reactor under a certain radiation intensity controlled by regulating the light source. Unless otherwise specified, the average radiation intensity of UV $(365 \mathrm{~nm})$ and visible light $(400 \sim 1,000 \mathrm{~nm})$, and the illuminance thereof on the surface of the dye solution are respectively $0.49 \mathrm{~mW} / \mathrm{cm}^{2}, 5.07 \mathrm{~mW} / \mathrm{cm}^{2}$ and $22.1 \times 10^{3} \mathrm{Lux}$.

Measure the absorbency of the dye solution at the maximum absorption wavelength with spectrophotometer at regular intervals, and calculate the decoloration rate with the following formula (3).

$$
D(\%)=\left(A_{0}-A\right) / A_{0} \times 100 \%
$$

Wherein, $A_{0}$ and $A$ respectively represent the initial absorbency and after-reaction absorbency of the dye solution at the maximum absorption wavelength.

\section{Result and discussion}

\subsection{Coordination reaction between $A O-P A N$ fiber and $\mathrm{Fe}^{3+}$}

\subsubsection{Effects of $C_{\mathrm{Fe}}$ on $C_{\mathrm{Fe}-\mathrm{AO}-\mathrm{PAN}}$}

Dip $1.0 \mathrm{~g}$ AO-PAN fibers $(C P \%=60.05 \%)$ into $\mathrm{FeCl}_{3}$ solutions of different initial concentrations, react at $20{ }^{\circ} \mathrm{C}$ for a given period to get Fe-AO-PAN catalysts. Figure 2 exhibits the relation between $C_{\mathrm{Fe}}$ and $C_{\mathrm{Fe}-\mathrm{AO}-\mathrm{PAN}}$.

Figure 2 indicates that $C_{\mathrm{Fe}-\mathrm{AO}-\mathrm{PAN}}$ increases with the reaction going on, reaches equilibrium after $120 \mathrm{~min}$, and is saturated after $300 \mathrm{~min}$. It is worth notice that $C_{\mathrm{Fe}-\mathrm{AO}-\mathrm{PAN}}$ increases with the rise of $C_{\mathrm{Fe}}$ at the equilibrium concentration. It is mainly because that the rise of $C_{\mathrm{Fe}}$ increases the contact probability between $\mathrm{Fe}^{3+}$ and the surface of AO-PAN fiber, 
and speeds up its diffusion into the fiber, in turn accelerates the coordination reaction of $\mathrm{Fe}^{3+}$ with amino nitrogen and hydroxyl oxygen in the amidoxime group, finally increases the amount of the fixed $\mathrm{Fe}^{3+}$ on the fiber.

\subsubsection{Effects of reaction temperature on $C_{\mathrm{Fe}-\mathrm{AO}-\mathrm{PAN}}$}

Dip $1.0 \mathrm{~g}$ AO-PAN fiber $(C P \%=60.05 \%)$ into $0.1 \mathrm{~mol} / \mathrm{L} \mathrm{FeCl}_{3}$ solution, react at different temperatures to get Fe-AO-PAN catalysts. Figure 3 exhibits the relation between the reaction temperature and $C_{\mathrm{Fe}-\mathrm{AO}-\mathrm{PAN}}$.

From figure 3 , we can see that $C_{\mathrm{Fe}-\mathrm{AO}-\mathrm{PAN}}$ increases with the rise of reaction temperature after the same reaction period. It indicates that the rise of reaction temperature helps the coordination reaction between the amidoxime group in AO-PAN fiber and $\mathrm{Fe}^{3+}$, which is probably due to the heat absorption during reaction. In addition, the swelling capacity of PAN fiber increases with the rise of temperature, which not only makes $\mathrm{Fe}^{3+}$ much easier to diffuse into the fiber, but also increases the exposure area of amidoxime group. As a result, the reaction probability between $\mathrm{Fe}^{3+}$ and amidoxime

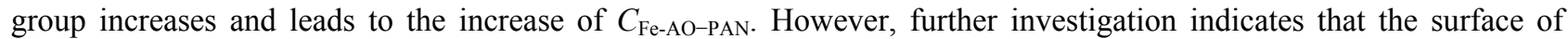
amidoxime fiber shrinkages and turns hard after coordinating with $\mathrm{Fe}^{3+}$, and its mechanical properties reduce, therefore, it is not proper to carry out the coordination reaction at a high temperature.

\subsection{Catalytic degradation of Fe-AO-PAN catalyst}

\subsubsection{Effects of $C_{\mathrm{Fe}-\mathrm{AO}-\mathrm{PAN}}$ on catalytic degradation}

Under the conditions of $\mathrm{pH} 6.0$ and radiation, add $1 \mathrm{~g}$ Fe-AO-PAN catalysts of different $C_{\mathrm{Fe}-\mathrm{AO}-\mathrm{PAN}}(0.00 \mathrm{mg} / \mathrm{g}, 30.60$ $\mathrm{mg} / \mathrm{g}, 40.46 \mathrm{mg} / \mathrm{g}, 52.80 \mathrm{mg} / \mathrm{g}$ and $62.78 \mathrm{mg} / \mathrm{g}$ ) respectively into Rhodamine B solutions, and catalytically degrade in the water-cooled photochemical reactor. Figure 4 exhibits the effects of $C_{\mathrm{Fe}-\mathrm{AO}-\mathrm{PAN}}$ on decoloration rate.

From figure 4, we can see that even though the decoloration rate increases with the reaction going on when $C_{\mathrm{Fe}-\mathrm{AO}-\mathrm{PAN}}$ is zero, it is still less than $15 \%$ after 90 minutes' reaction. This slow increase mainly comes from the limited absorption of the dye on PAN fiber. However, decoloration rate of the dye increases markedly when $C_{\mathrm{Fe}-\mathrm{AO}-\mathrm{PAN}}$ is up to $30.60 \mathrm{mg} / \mathrm{g}$, and continues to increase with the rise of $C_{\mathrm{Fe}-\mathrm{AO}-\mathrm{PAN}}$. This rapid increase is due to the catalysis resulted from the surface unsaturation of Fe-AO-PAN: the rise of $C_{\mathrm{Fe}-\mathrm{AO}-\mathrm{PAN}}$ accelerates $\mathrm{H}_{2} \mathrm{O}_{2}$ to decompose into $\mathrm{HO}$, and $\mathrm{HO}$ - can initiate the chain decomposition reaction, which increases the decoloration rate of the dye.

\subsubsection{Effects of radiation intensity on catalytic degradation}

Under the conditions of $\mathrm{pH} 6.0$ and in the presence of $0.50 \mathrm{~g}$ Fe-AO-PAN catalyst $\left(C_{\mathrm{Fe}-\mathrm{AO}-\mathrm{PAN}}=52.81 \mathrm{mg} / \mathrm{g}\right)$, the catalytic degradation of Rhodamine B is carried out at different radiation intensity in a water-cooled photochemical reactor (Table 1). Figure 5 exhibits the effects of radiation intensity on catalytic degradation.

From figure 5, we can see that the decoloration rate of Rhodamine B increases with the reaction going on, and almost reaches $70 \%$ after 70 minutes' reaction without radiation. It indicates that Fe-AO-PAN can catalyze the degradation of the dye even at dark state. However, the decoloration rate of Rhodamine B increases much sharper with the rise of radiation intensity. It indicates that the catalysis of Fe-AO-PAN to oxidation of the dye is closely related to the radiation intensity. This is probably because light radiation helps the dye molecular absorbed on the surface of catalyst to be excited to emit electron, and promotes the cyclic catalytic reaction between $\mathrm{Fe}^{3+}$ and $\mathrm{Fe}^{2+}$ to produce more $\mathrm{HO}$. $(\mathrm{Li}$, Jing, 2003, p. 2214-2215), in turn accelerates the degradation of the dye.

\subsubsection{Effects of dye concentration on catalytic degradation}

Under the conditions of $\mathrm{pH} 6.0$ and radiation, separately add $0.5 \mathrm{~g}$ Fe-AO-PAN catalysts $\left(C_{\mathrm{Fe}-\mathrm{AO}-\mathrm{PAN}}=28.56 \mathrm{mg} / \mathrm{g}\right)$ into Rhodamine B solutions of different concentrations. Figure 6 exhibits the effects of dye concentration on catalytic degradation.

From figure 6, we can see that the decoloration rate of Rhodamine B decreases with the rise of initial concentration of the dye after the same reaction period. This is probably related to the equilibrium between association and dissociation of the dye in water solution (Zhang, Zhuangyu, 1991, p. 81-82). Namely, association increases and dissociation decreases with the rise of initial concentration of the dye, and the contact probability of the dissociated dye with strong oxidant (HO-) reduces. As a result, the decoloration rate of the dye decreases.

\subsubsection{Effects of $\mathrm{pH}$ on catalytic degradation}

Under the condition of radiation and in the presence of $0.5 \mathrm{~g}$ Fe-AO-PAN catalysts $\left(C_{\mathrm{Fe}-\mathrm{AO}-\mathrm{PAN}}=28.56 \mathrm{mg} / \mathrm{g}\right)$, investigate the catalytic degradation of Rhodamine B solutions at different $\mathrm{pH}$. Figure 7 exhibits the effects of $\mathrm{pH}$ on catalytic degradation.

Figure 7 indicates that the decoloration rate of the dye increases with the rise of $\mathrm{pH}$ in an acid environment, and reaches the maximum at the condition of $\mathrm{pH} 6.0$, however decreases with the rise of $\mathrm{pH}$ in a basic environment. This is probably because proton can strongly capture HO- to slow down the degradation of the dye in an acid environment, especially in the solution of $\mathrm{pH}$ less than 3.0 (Zhang, Zhuangyu, 1991, p. 81-82). While in a basic environment, iron ion with catalytic activity is susceptible to hydrolysis to form FeOOH precipitation (Feng, J.Y., 2003, p. 2058-2066), and absorbs 
large amount of hydroxide ion to inhibit the catalytic activity of itself. In addition, the rise of $\mathrm{pH}$ not only affects the absorption of the dye on the surface of catalyst (Zhang, Zhuangyu, 1991, p. 81-82), but also causes the excessive decomposition of $\mathrm{H}_{2} \mathrm{O}_{2}$ (Zhang, Zhuangyu, 1991, p. 81-82), as a result, the amount of HO- reduces, and leads to the decrease of the decoloration rate of the dye.

\subsubsection{Reutilization of Fe-AO-PAN catalyst}

In order to investigate the service life of Fe-AO-PAN catalyst, take Fe-AO-PAN out of the solution after degradation $\left(C_{\mathrm{Fe}-\mathrm{AO}-\mathrm{PAN}}=52.81 \mathrm{mg} / \mathrm{g}\right)$, repeatedly wash with distilled water, and reuse in a new dye solution, again in a third new dye solution. Figure 8 exhibits the reutilization effect of Fe-AO-PAN catalyst in degrading Rhodamine $\mathrm{B}$.

Figure 8 indicates that the decoloration rate of Fe-AO-PAN catalyst in Rhodamine B solution after 40 minutes' reaction is up to $80 \%$ for the first use, lowers to $58 \%$ for the second use, and less than $40 \%$ for the third use. It proves that the catalytic activity of regenerated Fe-AO-PAN decreases. It can be explained in the following ways: on the one hand, Fe-AO-PAN catalyst is deactivated because its active center (iron ion) absorbs by-product, impurities and other catalyst poisons, and its catalysis on the degradation of the dye decreases or disappears; on the other hand, the surface of regenerated Fe-AO-PAN catalyst, blocked with impurities, can not contact the dye efficiently, and causes the decrease of decoloration rate.

\subsubsection{Analysis by UV-visible light spectrum}

The structure change of aromatic ring in Rhodamine B is investigated by analyzing its degradation procedure with UV-visible light spectrophotometer. The results are shown in figure 9.

Figure 9 indicates that two characteristic peaks $(255 \mathrm{~nm}$ and $550 \mathrm{~nm}$ ) of Rhodamine B become weaker with the reaction going on. By comparing figure 9 (a) with figure 9 (b), we can see that the characteristic peaks disappear much faster with the increase of $C_{\mathrm{Fe}-\mathrm{AO}-\mathrm{PAN}}$. It indicates that azo bond and aromatic ring in the dye molecular break in the presence

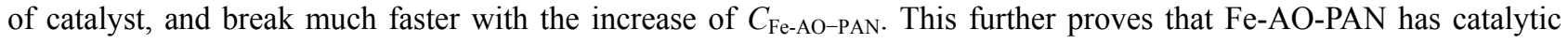
activity on both decoloration procedure of Rhodamine $\mathrm{B}$ and degradation procedure of molecular structure.

\section{Conclusion}

(1) Fe-AO-PAN catalyst is prepared with amidoxime modified polyacrylonitrile fiber (AO-PAN) and $\mathrm{FeCl}_{3}$. The content of active center (iron ion) on the surface of catalyst increases with the rise of iron ion concentration in solution, reaction temperature, as well as reaction time.

(2) Fe-AO-PAN catalyst has significant catalytic activity on the degradation of Rhodamine B, and this catalysis increases with the increase of $C_{\mathrm{Fe}-\mathrm{AO}-\mathrm{PAN} \text {. }}$

(3) The increase of the concentration of Rhodamine B solution may cause the decrease of decoloration rate. The catalytic activity of Fe-AO-PAN reaches the maximum at $\mathrm{pH}$ 6.0, and decreases whether $\mathrm{pH}$ increases or decreases.

(4) Even though Fe-AO-PAN has certain catalytic activity on the degradation of Rhodamine B without light radiation, the radiation intensity shows a significant positive correlation with the catalytic activity of Fe-AO-PAN.

(5) UV-visible light spectrum indicates that Fe-AO-PAN catalyst can effectively break the molecular structure of Rhodamine B, but its reutilization efficiency is not very high and needs further study.

\section{References}

Dong, Yongchun, Du, Fang, Ma, Hanxiao, Han, Zhenbang \& Cao, Yanan. (2008). Preparation of a Fe-modified polyacrylonitrile fibrous photocatalyst and its application in degradation of Reactive Red MS. The Chinese Journal of Process Engineering, 8(2), 359-365.

Feng, J.Y., Hu, X. J., Yue, P. L., Zhu, H. Y. \& Lu, G. Q. (2003). Degradation of Azo-Dye Orange II by a photoassisted Fenton reaction using a novel composite of iron oxide and silicate nano-particles as a catalyst. Industrial \& Engineering Chemistry Research, 42, 2058-2066.

Li, Jing, Ma, Wanhong, Huang, Yingping, Cheng, Mingming, Zhao, Jincai \& Yu, Jimmy C. (2003). A highly selective photooxidation approach using $\mathrm{O}_{2}$ in water catalyzed by iron (II) bipyridine complex supported on NaY zeolite. Chemical Communications, 2214-2215.

Tao, Tingxian, Wu, Zhichuan \& Zhao, Zeqing. (2001). Preparation of chelating fibers: modification of polyacrylonitrile fiber. Synthetic Fiber in China, 30(4), 32-42.

V. V. Ishtchenko, K. D. Huddersman \& R. F. Vitkovskaya. (2003). Production of a modified PAN fibrous catalyst and its optimisation towards the decomposition of hydrogen peroxide. Applied Catalysis, 242, 123-137.

Zhang, Zhuangyu \& Wu, Zuwang. (1991). Application of Dye. Beijing: Chemical Industry Press, p. 81-82. 
Table 1. Four different radiation intensities

\begin{tabular}{|c|c|c|c|}
\hline Radiation light & $\mathrm{UV}(365 \mathrm{~nm}) / \mathrm{mW} \cdot \mathrm{cm}^{-2}$ & Visible light $(400 \sim 1,000 \mathrm{~nm}) / \mathrm{mW} \cdot \mathrm{cm}^{-2}$ & Illuminance / Lux \\
\hline (A) & 0.00 & 0.001 & 1.8 \\
\hline (B) & 0.38 & 1.58 & $5.11 \times 10^{3}$ \\
\hline (C) & 0.49 & 5.07 & $22.1 \times 10^{3}$ \\
\hline (D) & 0.99 & 9.38 & $27.4 \times 10^{3}$ \\
\hline
\end{tabular}

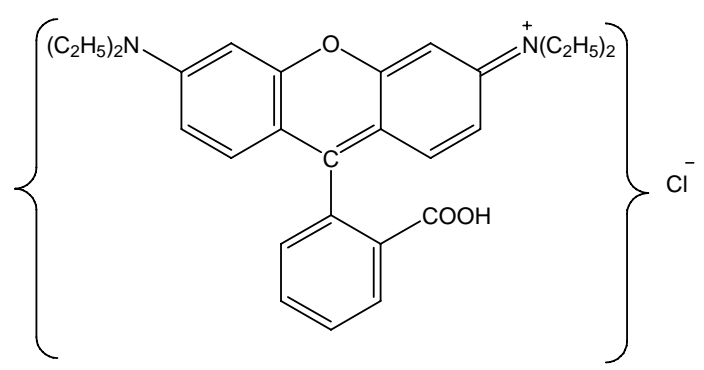

Figure 1. Chemical structure of Rhodamine B

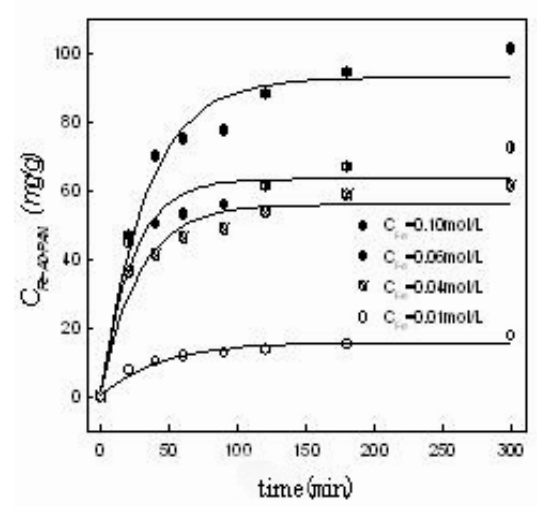

Figure 2. The effect of $C_{\mathrm{Fe}}$ on $C_{\mathrm{Fe}-\mathrm{AO}-\mathrm{PAN}}$

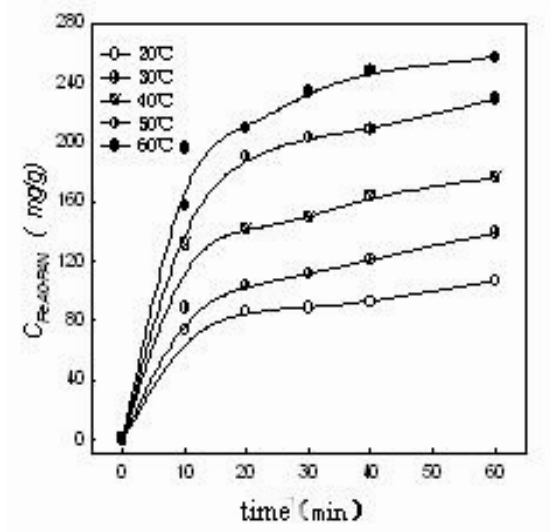

Figure 3. The effect of reaction temperature on $C_{\mathrm{Fe}-\mathrm{AO}-\mathrm{PAN}}$ 


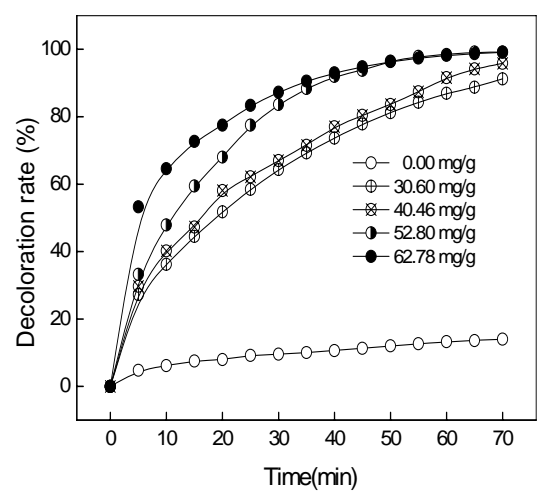

Figure 4. The effects of $C_{\mathrm{Fe}-\mathrm{AO}-\mathrm{PAN}}$ on catalytic degradation

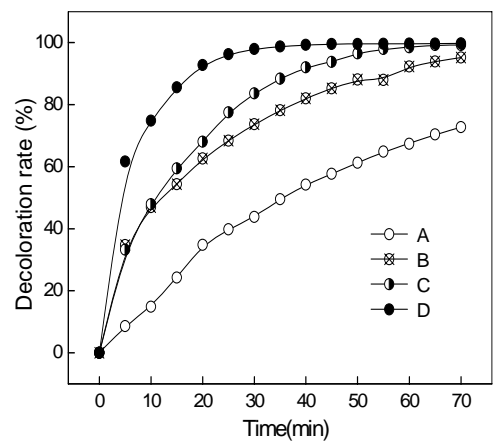

Figure 5 . The effects of radiation intensity on catalytic degradation

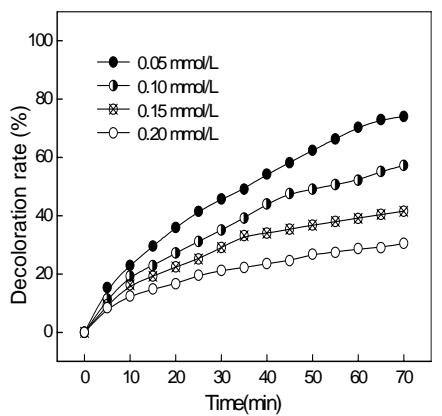

Figure 6 . The effects of dye concentration on catalytic degradation

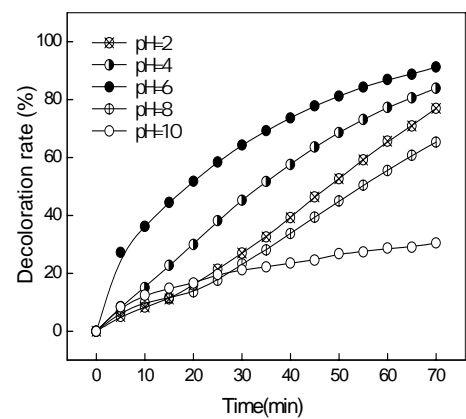

Figure 7. The effects of $\mathrm{pH}$ on catalytic degradation 


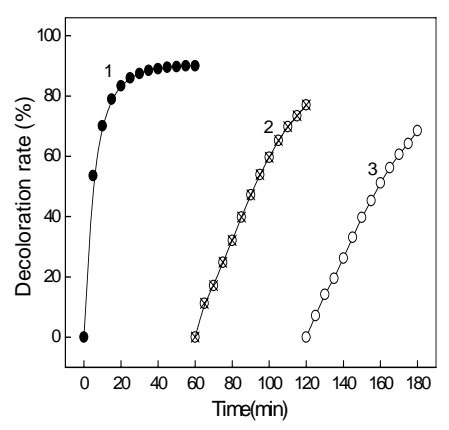

Figure 8. Reutilization of Fe-AO-PAN catalyst

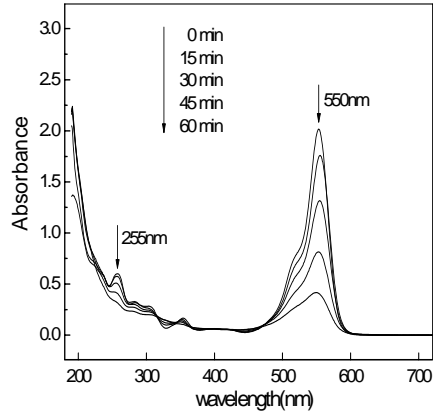

(a) $C_{\text {Fe-AO-PAN }}=28.56 \mathrm{mg} / \mathrm{g}$

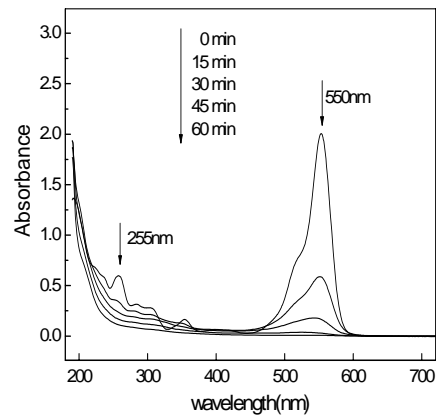

(b) $C_{\mathrm{Fe}-\mathrm{AO}-\mathrm{PAN}}=86.41 \mathrm{mg} / \mathrm{g}$

Figure 9. UV-visible light spectrum of degradation of Rhodamine B 